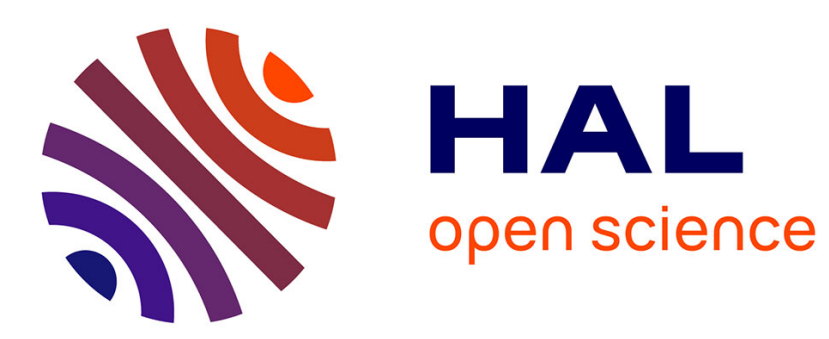

\title{
Diffusion de la lumière par les ondes de surface sur CO2 près du point critique mesure de la tension superficielle
}

J. Meunier

\section{To cite this version:}

J. Meunier. Diffusion de la lumière par les ondes de surface sur CO2 près du point critique mesure de la tension superficielle. Journal de Physique, 1969, 30 (11-12), pp.933-942. 10.1051/jphys:01969003001112093300 . jpa-00206861

\section{HAL Id: jpa-00206861 https://hal.science/jpa-00206861}

Submitted on 1 Jan 1969

HAL is a multi-disciplinary open access archive for the deposit and dissemination of scientific research documents, whether they are published or not. The documents may come from teaching and research institutions in France or abroad, or from public or private research centers.
L'archive ouverte pluridisciplinaire HAL, est destinée au dépôt et à la diffusion de documents scientifiques de niveau recherche, publiés ou non, émanant des établissements d'enseignement et de recherche français ou étrangers, des laboratoires publics ou privés. 


\title{
DIFFUSION DE LA LUMIÈRE PAR LES ONDES DE SURFAGE SUR $\mathrm{CO}_{2}$ PRÈS DU POINT GRITIQUE MESURE DE LA TENSION SUPERFIGIELLE
}

\author{
Par J. MEUNIER, \\ Faculté des Sciences de Paris, Laboratoire de Spectroscopie Hertzienne de l'E.N.S., \\ associé au C.N.R.S., 24, rue Lhomond, Paris, $5^{\mathrm{e}}$. \\ (Reçu le 3 juillet 1969.)
}

\begin{abstract}
Résumé. - L'analyse spectrale à haute résolution de la lumière diffusée par l'interface liquide-gaz de l'anhydride carbonique permet la mesure de la tension superficielle $\alpha$ à l'interface ; de l'écart de masse spécifique entre phases et de la viscosité. La tension superficielle a été mesurée dans le domaine de température $0,034 \leqslant T_{\mathrm{c}}-T \leqslant 31{ }^{\circ} \mathrm{C}$. Les résultats sont compatibles avec la relation $\alpha=\alpha_{0}\left(1-T / T_{\mathrm{e}}\right)^{\mu}$ avec $\mu=1,253 \pm 0,010$ et $\alpha_{0}=17,9 \pm 1,2 \mathrm{dynes} / \mathrm{cm}$ D'après les résultats expérimentaux, on peut conclure qu'il n'existe aucune anomalie de la viscosité dépassant $15 \%$ jusqu’à $0,034{ }^{\circ} \mathrm{C}$ du point critique.
\end{abstract}

Abstract. - Spectrum analysis with high resolution of the light scattered from the liquidgas interface of $\mathrm{CO}_{2}$ allows to measure the interfacial tension $\alpha$, the difference between the specific masses of the two phases and the viscosity. The surface tension has been measured in the temperature range $0.034 \leqslant T_{\mathrm{c}}-T \leqslant 31^{\circ} \mathrm{C}$. The results are well represented by $\alpha=\alpha_{0}\left(1-T / T_{\mathrm{e}}\right)^{\mu}$, with $\mu=1.253 \pm 0.010$ and $\alpha_{0}=17.9 \pm 1.2 \mathrm{dynes} / \mathrm{cm}$. No anomaly

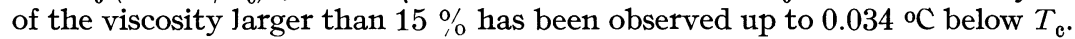

I. Introduction. - Les fluctuations thermiques produisent à l'interface liquide-gaz d'un fluide à l'équilibre des aspérités de très faibles amplitudes dont l'évolution temporelle est décrite par l'hydrodynamique. Ces fluctuations peuvent être étudiées par diffusion inélastique de lumière cohérente. De nombreux résultats ont déjà été obtenus dans le cas de l'interface d'un liquide et de l'air [1], [2]. Nous décrivons ici l'étude systématique du spectre de la lumière diffusée dans plusieurs directions proches de la réflexion spéculaire par l'anhydride carbonique près du point critique et nous analysons les résultats en fonction de $T_{\mathrm{c}}-T$. L'expérience est réalisable grâce à l'existence d'un phénomène d'opalescence de surface qui fait que la diffusion de surface reste très supérieure à la diffusion en volume tant que l'épaisseur de l'interface est très inférieure à la longueur d'onde. Ce phénomène a été observé pour la première fois par Mandelstam [9] et analysé récemment par Papoular [3] dans le cas d'un mélange critique de démixtion. Les conclusions restent valables pour un point critique de gaz pur.

L'anhydride carbonique est un liquide très peu visqueux. Loin du point critique $\left(0,2 \leqslant T_{\mathrm{c}}-T \leqslant 31^{\circ} \mathrm{C}\right)$, les fluctuations de surface sont des ondes de capillarité très peu amorties. L'étude de leur fréquence permet de déterminer la tension superficielle à l'interface. Plus près du point critique $\left(0,024 \leqslant T_{\mathrm{c}}-T \leqslant 0,2^{\circ} \mathrm{C}\right)$, la tension superficielle devient très faible. Elle tend vers zéro avec $T_{\mathrm{c}}-T$ plus rapidement que la différence de masse spécifique entre les deux phases, aussi la gravité se met à intervenir notablement dans la propagation des ondes de surface. La gravité joue un rôle d'autant plus grand, à température fixée, que le vecteur d'onde $q$ de l'onde (et par suite l'angle de diffusion) est plus petit. L'étude spectrale pour plusieurs valeurs de $q$ permet à la fois de déterminer la tension superficielle et l'écart de masse spécifique entre phases. L'amortissement des ondes est très important près de $T_{\mathrm{c}}$ par suite de la valeur très faible des forces de rappel (gravité et capillarité). La mesure de leur durée de vie permet de déterminer la viscosité.

L'étude théorique des fluctuations à l'interface liquide-gaz est faite au chapitre II. Le montage expérimental est décrit au chapitre III et les résultats expérimentaux sont donnés et interprétés au chapitre IV.

II. Fonction de corrélation et loi de dispersion des fluctuations à l'interface liquide-gaz. - 1 . FONCTION DE GORRÉLATION DES FLUGTUATIONS DE SURFACE. Nous désignerons par $\rho, \eta$ la masse spécifique et la viscosité du liquide; $\rho^{\prime}, \eta^{\prime}$ celles du gaz; $\alpha$ la tension superficielle à l'interface. $\Delta \rho=\rho-\rho^{\prime}$ est l'écart de 
masse spécifique et $\Delta \eta=\eta-\eta^{\prime}$ est l'écart de viscosité entre phases. Nous utiliserons pour simplifier l'écriture le paramètre sans dimension :

$$
y=\frac{\alpha_{q} \cdot\left(\rho+\rho^{\prime}\right)}{4\left(\eta+\eta^{\prime}\right)^{2} q}
$$

(dans lequel $\alpha_{q}=\alpha+\frac{\Delta \rho \cdot g}{q^{2}}$ et $g$ est l'accélération de la pesanteur) et la constante de temps :

$$
\tau_{0}=\frac{\rho+\rho^{\prime}}{2\left(\eta+\eta^{\prime}\right) q^{2}} .
$$

Si $\omega=2 \pi \nu$ est la pulsation d'une onde, $\gamma=\pi \Delta \nu$ son amortissement, nous désignerons par $S$ le paramètre sans dimension suivant :

$$
S=S_{r}+i S_{i} \text { avec } S_{r}=\gamma \tau_{0} ; \quad S_{i}=\omega \tau_{0} .
$$

Soit $\zeta(\mathbf{r}, t)$ le déplacement vertical au temps $t$ de l'élément de l'interface dont la projection sur le plan horizontal est définie par $\mathbf{r}$. On montre [4] que la fonction de corrélation $g(R, \tau)=\overline{\zeta(\mathbf{r}+\mathbf{R}, t+\tau) \zeta(\mathbf{r}, t)}$ ne dépend que de $R$ et $\tau$ et peut se mettre sous la forme d'une somme de fonctions de corrélation $G_{q}(\tau)$ :

$$
g(R, \tau)=A(2 \pi)^{-2} \int G_{q}(\tau) \exp (i \mathbf{q} \cdot \mathbf{R}) \mathrm{d}^{2} q
$$

$A$ est l'aire de l'interface; $G_{q}(\tau)$ est la fonction de corrélation des fluctuations de vecteur d'onde $q$.

Lesfluctuations thermiques observées ont des amplitudes très faibles devant leur longueur d'onde, par suite les conditions de linéarisation des équations de l'hydrodynamique sont satisfaites et les fonctions $G_{q}(\tau)$ sont indépendantes. On montre alors que [4] :

$$
\begin{aligned}
G_{q}(\tau)=\frac{k T}{\alpha_{q} q^{2} A}\left(S_{2 q}-S_{1 q}\right)^{-1} & {\left[S_{2 q} \exp \left(S_{1 q}\left|\frac{\tau}{\tau_{0}}\right|\right)\right.} \\
& \left.-S_{1 q} \exp \left(S_{2 q}\left|\frac{\tau}{\tau_{0}}\right|\right)\right]
\end{aligned}
$$

$S_{1 q}$ et $S_{2 q}$ sont les deux solutions de l'équation de dispersion des ondes libres à l'interface, de vecteur d'onde $q$ et d'évolution temporelle $\exp (i \mathbf{q} \mathbf{r}) \exp \left(S \frac{t}{\tau_{0}}\right)$.

2. ÉQuATION DE DISPERSION DES ONDES LibRES A L'INTERFAGE. - L'hydrodynamique permet de calculer l'équation de dispersion des ondes libres à l'interface. Nous supposerons le fluide incompressible. Cette hypothèse est justifiée en appendice. L'équation de dispersion s'écrit alors [5] :

$$
D_{3}=\left|\begin{array}{ccc}
2\left(\eta+\eta^{\prime}\right) q & 2 \eta^{\prime} q+\eta\left(m+\frac{q^{2}}{m}\right) & 2 \eta q+\eta^{\prime}\left(m^{\prime}+\frac{q^{2}}{m^{\prime}}\right) \\
2 \Delta \eta \Omega q+i\left(\alpha_{q} q^{2}+\frac{\Omega^{2}}{q} \Delta \rho\right) & 2 \Delta \eta \Omega q+i\left(\alpha_{q} \frac{q^{3}}{m}+\Omega^{2} \frac{\rho^{\prime}}{q}\right) & 2 \Delta \eta \Omega q+i\left(\alpha_{q} q^{2}-\frac{\Omega^{2}}{q} \rho\right) \\
2 & \frac{q}{m}+1 & \frac{q}{m^{\prime}}+1
\end{array}\right|
$$$$
=0
$$

dans laquelle :

$$
\begin{aligned}
& \Omega=\omega-i \gamma \\
& m^{2}=q^{2}-i \frac{\Omega \rho}{\eta} \quad \text { avec } R(m)>0 \text { (partie réelle) } \\
& m^{\prime 2}=q^{2}-i \Omega \frac{\rho^{\prime}}{\eta^{\prime}} \quad \text { avec } R\left(m^{\prime}\right)>0 .
\end{aligned}
$$

L'équation (1) a pour chaque valeur de $q$ deux solutions $S_{1 q}$ et $S_{2 q}$ physiquement acceptables, c'est-àdire correspondant à des ondes amorties.

Il existe une valeur critique du paramètre $y$ $\left(y_{0} \approx 0,15\right)$ telle que si $y<y_{0}$ les fluctuations de surface s'amortissent sans se propager : $S_{1 q}$ et $S_{2 q}$ sont réels, si $y>y_{0}$, elles se propagent en s'amortissant : $S_{1 q}$ et $S_{2 q}$ sont complexes. Les mesures expérimentales sur l'anhydride carbonique ont toujours été faites sur des ondes de capillarité en régime oscillant, c'est-à-dire $y>y_{0}$, et par suite :

$$
S_{1 q}=S_{r}+i S_{i} ; \quad S_{2 q}=S_{r}-i S_{i} .
$$

La transformée de Fourier de $G_{q}(\tau)$ s'écrit alors :

$$
\begin{aligned}
P_{q}(\omega)= & \frac{k T}{\alpha_{q} q^{2} A} \tau_{0} \frac{S_{r}^{2}+S_{i}^{2}}{S_{i}^{2}}\left\{\frac{S_{r}}{S_{r}^{2}+\left(\omega \tau_{0}+S_{i}\right)^{2}}\right. \\
+\frac{S_{r}}{S_{r}^{2}+\left(\omega \tau_{0}-S_{i}\right)^{2}} & +\frac{S_{r}}{S_{i}}\left[\frac{S_{i}-\omega \tau_{0}}{S_{r}^{2}+\left(\omega \tau_{0}-S_{i}\right)^{2}}\right. \\
& \left.\left.+\frac{S_{i}+\omega \tau_{0}}{S_{r}^{2}+\left(\omega \tau_{0}+S_{i}\right)^{2}}\right]\right\}
\end{aligned}
$$

$P_{q}(\omega)$ est le spectre des fluctuations thermiques de vecteur d'onde $q$. Ce spectre présente un maximum pour la fréquence $\omega^{(\mathrm{a})}$ telle que :

$$
\omega^{(\mathrm{a})} \tau_{0}=\sqrt{S_{i}^{2}-S_{r}^{2}}
$$

on posera :

$$
S_{i}^{(\mathrm{a})}=\omega^{(\mathrm{a})} \tau_{0}=2 \pi \nu^{(\mathrm{a})} \tau_{0} .
$$


3. Simplification $\eta / \rho=\eta^{\prime} / \rho^{\prime}$. - L'équation de dispersion des ondes de capillarité (1) contient les paramètres $\rho, \rho^{\prime}, \eta, \eta^{\prime}$. Afin de simplifier celle-ci, nous allons analyser les résultats expérimentaux de la viscosité de l'anhydride carbonique en fonction de la masse spécifique et de la température.

La viscosité de l'anhydride carbonique a été étudiée par des méthodes classiques. Les résultats expérimentaux de la mesure de $\eta$ en fonction de $\rho$ le long de l'isotherme critique [6], [7] $\left(T=31,1^{\circ} \mathrm{C}\right)$ sont reproduits figure 1 . Cette courbe présente une légère

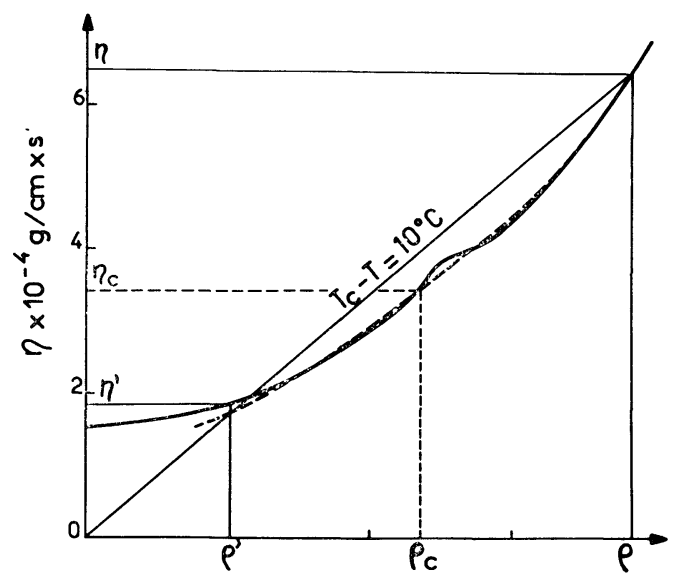

FIG. 1. - Viscosité de $\mathrm{CO}_{2}$ en fonction de la masse spécifique le long de l'isotherme critique d'après la référence [7]. En pointillés, la courbe $\eta_{1}(\rho)$ utilisée dans l'approximation $\eta / p=\eta^{\prime} / \rho^{\prime}$.

anomalie ( $6 \%$ environ) au voisinage de la densité critique $\rho=\rho_{c}$. Il est difficile de dire si cette anomalie est réelle ou provient d'une insuffisance de la correction à apporter aux mesures par suite de la grande compressibilité du fluide dans cette zone. Elle devient en effet d'autant plus faible que la précision des mesures est meilleure [6], [7]. Si nous négligeons cette faible anomalie, les résultats de la référence [7] montrent que la variation de $\eta(\rho)$ en fonction de la température est très faible. Par exemple, sur l'intervalle de température $T-T_{\mathrm{c}}<19{ }^{\circ} \mathrm{C}, \eta(\rho)$ ne varie que de $3 \%$ pour $\rho_{\mathrm{c}}<\rho<2 \rho_{\mathrm{c}}$ et $6 \%$ pour $\rho<\rho_{\mathrm{c}}$. Nous ne disposons que de mesures faites au-dessus du point critique [7] et nous admettrons que cette propriété demeure au-dessous de $T_{\mathrm{c}}$. On constate du reste qu'elle est vérifiée par un grand nombre de fluides simples [13]. Moyennant les approximations qui précèdent, la figure 1 permet de déterminer la viscosité $\eta$ du liquide anhydride carbonique et celle $\eta^{\prime}$ de sa vapeur le long de la courbe de saturation. Nous constatons alors que la relation :

$$
\frac{\eta}{\rho}=\frac{\eta^{\prime}}{\rho^{\prime}}
$$

se trouve satisfaite avec une assez bonne approximation. Sur la figure 1 nous avons indiqué les valeurs de $\eta, \eta^{\prime}, \rho$, $\rho^{\prime}$ correspondant à $T_{\mathrm{c}}-T=10^{\circ} \mathrm{C}$. La correction à apporter à $\eta^{\prime}$ pour que l'égalité précédente soit satisfaite est inférieure à $10 \%$. La précision s'améliore si nous approchons du point critique. Lorsque $\rho \rightarrow \rho_{c}$ et $\rho^{\prime} \rightarrow \rho_{c}$, le rapport $\eta / \rho=\eta^{\prime} / \rho^{\prime}$ tend vers une valeur minimum que nous noterons $\eta_{\mathrm{c}} / \rho_{\mathrm{c}}$. Nous avons admis la valeur $\eta_{\mathrm{c}}=350 \times 10^{-6}$ poise avec $\rho_{\mathrm{c}}=0,464 \mathrm{~g} / \mathrm{cm}^{3}$. Cette valeur de $\eta_{c}$ est, comme nous le verrons, en bon accord avec nos résultats expérimentaux. Nous admettrons donc dans la suite la propriété $\eta / \rho=\eta^{\prime} / \rho^{\prime}$ avec pour valeur limite $\eta_{\mathrm{c}} / \rho_{\mathrm{c}}=(0,350 / 0,464) \times 10^{-3}$ G.G.S., ce qui revient à utiliser pour $\eta(\rho)$ le long de la courbe de saturation la courbe en pointillé de la figure 1. Avec cette approximation, l'équation de dispersion (1) des ondes à l'interface se réduit à :

$$
\begin{array}{r}
y=-\frac{2 \rho \rho^{\prime}}{\left(\rho+\rho^{\prime}\right)^{2}} S \sqrt{1+2 S}(1+\sqrt{1+2 S}) \\
-\frac{\left(\rho-\rho^{\prime}\right)^{2}}{\left(\rho+\rho^{\prime}\right)^{2}}\left[(1+S)^{2}-\sqrt{1+2 S}\right] .
\end{array}
$$

Notons que pour $\rho^{\prime}=0$ l'équation (3) redonne bien l'équation de dispersion des ondes à la surface libre d'un liquide :

$$
y=-(1+S)^{2}+\sqrt{1+2 S}
$$

tandis que pour $\rho=\rho^{\prime}$ l'équation (3) devient :

$$
y=-\frac{S}{2} \sqrt{1+2 S}(1+\sqrt{1+2 S}) .
$$

C'est l'équation de dispersion des ondes à l'interface séparant deux fluides de même densité et même viscosité.

4. Estimation Des erreurs. - Nous allons évaluer l'erreur commise en remplaçant l'équation de dispersion (1) par l'équation (3). Il est utile de distinguer deux zones de température, assez loin, puis près de $T_{\mathrm{c}}$.

1) Assez loin du point critique, le régime des ondes est très peu amorti : $S_{r} / S_{i} \ll 1$ ou $1 / S_{i} \ll 1$. L'équation (1) peut être remplacée par son développement limité au $1^{\text {er }}$ ordre en $1 / \sqrt{S_{i}}$ :

$$
\begin{aligned}
& \frac{4\left(\eta+\eta^{\prime}\right)^{2} q}{\rho+\rho^{\prime}} y=\alpha_{q} \\
& \quad=\left(\rho+\rho^{\prime}\right) \frac{\omega^{2}}{q^{3}}\left(1+\frac{2 \sqrt{\frac{\rho^{\prime}}{\rho}}}{\sqrt{\frac{\eta}{\eta^{\prime}}}+\sqrt{\frac{\rho^{\prime}}{\rho}}} \frac{1}{\sqrt{S_{i}}}+\ldots\right) .
\end{aligned}
$$

L'erreur commise sur $\eta$ et $\eta^{\prime}$ n'intervient pas sur le terme principal. L'erreur sur $\alpha_{q}$ est donc très faible : $\left(T_{\mathrm{c}}-T\right)=31^{\circ} \mathrm{C}$; le terme du $1 \mathrm{er}$ ordre représente $1 \%$ de $\alpha_{q}$. L'incertitude sur $\eta, \eta^{\prime}$ entraîne une incertitude négligeable sur $\alpha_{q} \cdot\left(T_{\mathrm{c}}-T\right)=0,5^{\circ} \mathrm{C}$; le terme du $1^{\text {er }}$ ordre représente moins de $40 \%$ de $\alpha_{q}$. Avec $3 \%$ d'erreur sur $\eta^{\prime}$ et $3 \%$ sur $\eta$, l'erreur sur le terme du $1^{\text {er }}$ ordre est de $3 \%$ et l'incertitude sur $\alpha_{q}$ est inférieure à $1,5 \%$. 
2) Au voisinage du point critique, $\Delta \rho / \rho \ll 1$. Nous nous limiterons au $1^{\text {er }}$ ordre en $\Delta \rho / \rho$. $\eta / \rho=\eta^{\prime} / \rho^{\prime}$ prend sa valeur minimum $\eta_{\mathrm{c}} / \rho_{\mathrm{c}}$. Dans cette approximation, l'équation de dispersion devient :

$y_{\mathrm{c}}=\frac{\alpha_{q} \rho_{\mathrm{c}}}{8 \eta_{\mathrm{c}}^{2} q}=-\frac{S}{2} \sqrt{1+2 S}(1+\sqrt{1+2 S})$

qui est l'équation de dispersion des ondes à l'interface de deux fluides ayant même viscosité $\eta_{\mathrm{c}}$ et même masse spécifique $\rho_{c}$. Sur la figure 2 nous avons tracé en

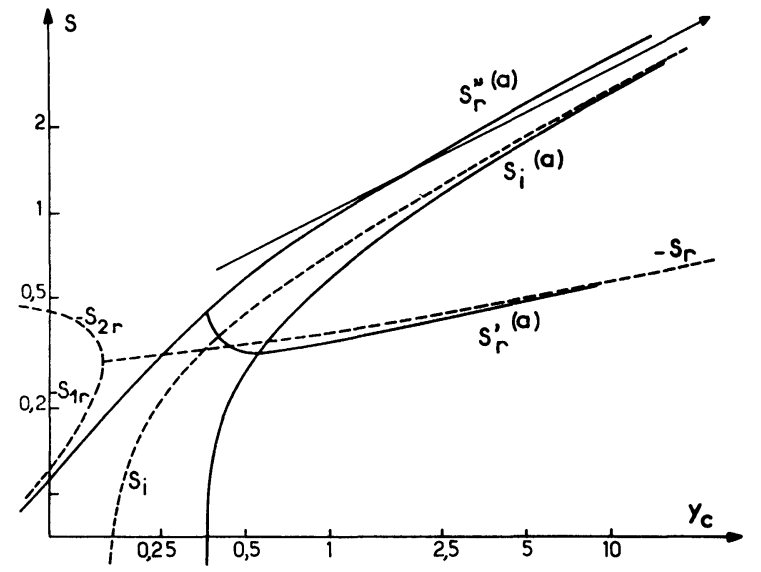

FIG. 2. - Courbes de dispersion des ondes à l'interface séparant deux fluides de même masse spécifique et même viscosité.

pointillé $S_{\imath}$ et $S_{r}$ en fonction de $y_{\mathrm{c}}$ et en trait plein les valeurs correspondantes de :

$$
S_{i}^{(\mathrm{a})}=2 \pi \nu^{(\mathrm{a})} \tau_{0}
$$

où $v^{(a)}$ est la fréquence du maximum du spectre $P_{q}(\nu)$ et :

$$
S_{r}^{\prime(\mathrm{a})}=\pi \Delta \nu^{(\mathrm{a})} \tau_{0} ; \quad S_{r}^{\prime \prime(\mathrm{a})}=S_{r}^{\prime(\mathrm{a})}+S_{i}^{(\mathrm{a})}
$$

où $\Delta v^{(\text {a) }}$ est la largeur à mi-hauteur de $P_{q}(\nu)$.

L'incertitude sur $\alpha_{q}$ due à l'erreur commise sur $\eta_{c}$ est :

$$
\frac{\Delta \alpha_{q}}{\alpha_{q}}=\left(2-\frac{\mathrm{d} y_{\mathrm{c}}}{\mathrm{d} S} \frac{S}{y_{\mathrm{c}}} \frac{\Delta \eta_{\mathrm{c}}}{\eta_{\mathrm{c}}}\right) .
$$

Pour $T_{\mathrm{c}}-T=0,034^{\circ} \mathrm{C}, q=383 \mathrm{~cm}^{-1}$ :

$$
\frac{\Delta \alpha_{q}}{\alpha_{q}} \approx \frac{1}{2} \frac{\Delta \eta_{\mathrm{c}}}{\eta_{\mathrm{c}}} \text {. }
$$

Cette valeur de $T_{\mathrm{c}}-T$ correspond au cas le plus défavorable. Si nous admettons la valeur donnée par la courbe $1: 350 \times 10^{-6}$ poise avec $4 \%$ d'erreur, l'incertitude sur $\alpha_{q}$ est de $2 \%$. Si nous utilisons par contre la valeur résultant de nos mesures (chap. IV) : $350 \pm 50 \times 10^{-6}$ poise, l'incertitude est de $3,5 \%$.

III. Diffusion de la lumière par les fluctuations thermiques à l'interface; montage expérimental. L'interface du point de vue de l'optique se comporte comme une superposition de réseaux de phase de périodicité $2 \pi / q$. Éclairé par un faisceau lumineux, chaque réseau donne en plus de la réflexion régulière un ensemble de spectres dont seuls ceux du premier ordre ont une intensité non négligeable par suite de la faible amplitude des fluctuations. Une direction d'observation quelconque coïncide toujours avec le maximum d'intensité diffusée par l'un des réseaux présents sur la surface. Ceci établit une relation univoque $q=\Delta \theta k \cos \theta \quad$ (fig. 3 ) entre l'angle de diffu-

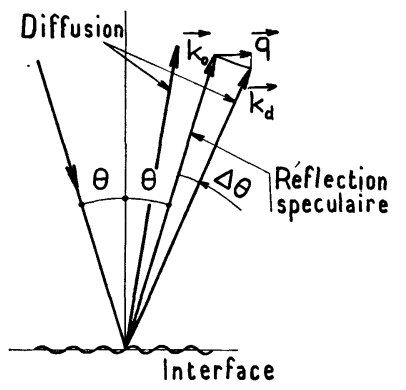

Fig. 3. - Schéma de la diffusion par l'interface liquidegaz : $k_{0}$ est le vecteur d'onde de la lumière incidente ; $k_{\mathrm{d}}$, celui de la lumière diffusée, et $q$ celui des ondes de surface.

sion $\Delta \theta$ et le vecteur d'onde $q$ des fluctuations responsables de la diffusion observée ( $\theta$ est l'angle d'incidence et $k_{0}$ le vecteur d'onde de la lumière). L'intensité diffusée [8], [9], [10] n'est importante qu'au voisinage de la réflexion régulière. L'incidence utilisée est proche de la normale.

La lumière incidente, de fréquence $v_{0}$, est diffusée sur des ondes de vecteur d'onde $q$ en mouvement. Le spectre de la lumière diffusée par celles-ci est identique au spectre $P_{q}(v)$ translaté de $\nu_{0}$ dans l'échelle des fréquences. Le montage expérimental a été décrit par ailleurs [11]. L'analyse spectrale est réalisée par la méthode hétérodyne et fournit un signal (densité spectrale du courant photoélectrique) proportionnel à $P_{q}(v)$. La résolution en fréquence est de $3 \mathrm{~Hz}$. L'angle $\Delta \theta$ de la direction d'observation et de la réflexion régulière est petit : $5,5^{\prime} \leqslant \Delta \theta \leqslant 55^{\prime}$, ce qui nous permet d'observer des ondes de vecteur d'onde $150 \leqslant q \leqslant 1500 \mathrm{~cm}^{-1}$.

Le faisceau laser (raie $6328 \AA$ d'un laser $\mathrm{He}-\mathrm{Ne}$ ) après filtrage spatial a une puissance d'environ $50 \mathrm{~mW}$. Loin du point critique $\left(5\right.$ à $\left.30^{\circ} \mathrm{C}\right)$, le montage est exactement celui que nous avons précédemment décrit [11]. Toute l'intensité lumineuse est utilisée et le diamètre du faisceau au niveau de l'interface est de $5 \mathrm{~mm}$ (soit une puissance par unité de surface de $250 \mathrm{~mW} / \mathrm{cm}^{2}$ ). Plus près du point critique, cette puissance est trop grande et provoque par échauffement des mouvements de convection qui déforment la surface. Nous avons dû diminuer l'intensité du faisceau par des facteurs 3 , 10,30 ou 100 selon les cas. Très près du point critique (quelque $10^{-1} \mathrm{o} \mathrm{C}$ ), la tension superficielle est très faible; l'interface est plane jusqu'à une très faible distance des parois et nous avons pu augmenter le dia- 
mètre du faisceau ( $18 \mathrm{~mm}$ ), ce qui permet de conserver une bonne homogénéité de température dans la cellule avec des puissances lumineuses par unité de surface relativement plus fortes. Très près du point critique, celle-ci était réduite à $0,2 \mathrm{~mW} / \mathrm{cm}^{2}$. Malgré cette atténuation considérable de l'intensité, le rapport signal sur bruit a très peu diminué par suite de l'augmentation de la diffusion (opalescence de surface).

L'anhydride carbonique est contenu dans une cellule en acier inoxydable de $2 \mathrm{~cm}$ de diamètre intérieur et $7 \mathrm{~mm}$ de hauteur, munie de deux fenêtres de quartz (diamètre utile, $2 \mathrm{~cm}$ ). Le remplissage est fait à la densité critique. Pour cela, la cellule est maintenue à $10^{\circ} \mathrm{C}$ et reliée par un tube très fin en cuivre recuit à une bouteille d'anhydride carbonique liquide de très haute pureté $(99,998 \%)$. L'anhydride carbonique distille lentement dans la cellule. Lorsque la densité moyenne de fluide dans la cellule est légèrement inférieure à la densité critique (moins de $45,7 \%$ de liquide à $10^{\circ} \mathrm{C}$ ), le tube de cuivre est coupé et soudé à quelques centimètres de la cellule. Celle-ci est alors placée dans un bain thermostaté à $30^{\circ} \mathrm{C}$ environ dont on monte la température jusqu'à $T_{\mathrm{c}}$ très lentement de manière à maintenir l'anhydride carbonique à l'équilibre thermodynamique. La densité étant inférieure à la densité critique, le ménisque descend. On écrase alors la portion du tube de cuivre encore attachée à la cellule afin de diminuer le volume occupé par le fluide et donc d'établir la densité critique $(50 \%$ de liquide à $T_{\mathrm{c}}=31,1^{\circ} \mathrm{G}$ lorsque le ménisque disparaît).

La cellule ainsi remplie est placée dans un thermostat qui comporte deux enceintes. Une enceinte externe $\mathrm{E}$ est thermostatée avec une assez bonne précision à l'aide d'un thermomètre à contact gradué au dizième de degré. Les dérives à long terme sont de $\pm 0,02^{\circ} \mathrm{C}$. Une enceinte interne $\mathrm{I}$, dans laquelle est placée la cellule, est thermostatée avec grande précision à l'aide d'un thermomètre à résistance de platine (dérives à long terme : $\pm 0,0005^{\circ} \mathrm{C}$ pour 5 jours). L'enceinte I est maintenue à une température légèrement inférieure $\left(0,1^{\circ} \mathrm{C}\right)$ à celle de $\mathbf{E}$ par un très faible courant d'air à $20^{\circ} \mathrm{C}$ circulant dans une tuyauterie soudée à la paroi. Ceci permet d'éviter toute condensation de l'anhydride carbonique sur la fenêtre supérieure de la cellule à travers laquelle sont faites les observations. L'isolement thermique entre les deux enceintes est très bon : la constante de temps d'équilibre de l'enceinte intérieure est de $5 \mathrm{~h}$.

IV. Résultats expérimentaux. - Nous allons voir que l'étude spectrale de la lumière diffusée sous plusieurs angles permet de déterminer $\nu_{a}(q, T)$ (la fréquence du maximum du spectre) et aussi $\Delta v_{a}(q, T)$ dans un nombre limité de cas que nous analysons au $\S$ A suivant. C'est à partir de ces mesures de fréquences que nous déduisons la tension superficielle et éventuellement la différence de masse spécifique entre phases et la viscosité $\eta_{\mathrm{c}}$ suivant la méthode décrite au $\S \mathrm{B}$. Les résultats sont indiqués au $\S \mathrm{C}$ où l'on donne aussi une estimation des différentes incertitudes et des causes essentielles qui limitent le domaine de température exploré.

A) Pour chaque valeur de la température, nous avons étudié les spectres expérimentaux pour différentes valeurs de $q: 150 \leqslant q \leqslant 1500 \mathrm{~cm}^{-1}$. La valeur du vecteur d'onde $q_{0}$ sélectionné est proportionnelle au diamètre $\Phi$ du diaphragme annulaire. En fait, la fente de ce diaphragme a une largeur $\Delta \Phi / 2$ et on sélectionne non pas un vecteur d'onde $q_{0}$ mais un ensemble de vecteurs d'ondes compris entre deux valeurs $q_{0} \pm \Delta q / 2$ avec $\Delta \Phi / \Phi=\Delta q / q_{0}$. Le diamètre de la tache de diffraction du faisceau est dans nos expériences négligeable devant la largeur $\Delta \Phi / 2$ de la fente. Le spectre expérimental tracé n'est donc pas le spectre $P_{q}(v)$ pour $q=q_{0}$, mais une superposition de spectres $P_{q}(\nu)$ pour des valeurs de $q$ comprises entre $q_{0}-\Delta q / 2$ et $q_{0}+\Delta q / 2$. La fréquence du maximum du spectre résultant correspond à la fréquence maximale de $P_{q}(\nu)$ pour la valeur moyenne $q=q_{0}$ du vecteur d'onde, tandis que la largeur à mi-hauteur n'a de sens que si elle est très supérieure au déplacement de fréquence $\delta \nu$ du maximum de $P_{q}(\nu)$ pour une variation $\Delta q$ de $q$. Pour $y \gg y_{0}$, la loi de dispersion des ondes de capillarité est en première approximation $\omega^{2}=\left(\alpha_{\alpha} / \rho+\rho^{\prime}\right) q^{3}$. Nous ne pourrons donc mesurer la demi-largeur naturelle $\Delta v_{a}$ du spectre $P_{q}(\nu)$ que si :

$$
\frac{\Delta v_{a}}{v_{a}} \gg \frac{\delta v}{v_{a}} \approx \frac{\Delta q}{q}=\frac{\Delta \Phi}{\Phi}
$$

Avec $\Phi=10 \mathrm{~mm}, \Delta \Phi=1 \mathrm{~mm}$ et $\Delta v_{a} / \delta \nu>10$, ceci nécessite $y<1,5$ ou $\Delta v_{a} / v_{a}>1$. Si l'on augmente $\Phi, \Delta \Phi / \Phi$ diminue, mais le rapport signal sur bruit décroît et on n'améliore pas la résolution du spectromètre. En conclusion, nous n'avons pu mesurer avec assez de précision l'amortissement des ondes de capillarité que lorsqu'il est important $\left(\Delta v_{a} / v_{a}>1\right.$, soit environ $y<1,5)$.

Pour des températures éloignées du point critique $0,1 \leqslant T_{\mathrm{c}}-T \leqslant 31^{\circ} \mathrm{C}$, seule la fréquence $\nu^{(\mathrm{a})} \mathrm{du}$ maximum des spectres $P_{q}(v)$ a été mesurée expérimentalement.

Pour $T_{\mathrm{c}}-T<0,1^{\circ} \mathrm{C}, \nu^{(\mathrm{a})}$ et $\Delta v^{(\mathrm{a})}$ ont été déterminés expérimentalement pour les plus grandes valeurs de $q($ fig. $4 \mathrm{c})$, tandis que $v^{(a)}$ seul a été déterminé pour les plus petites valeurs (fig. 4 a et $4 \mathrm{~b}$ ).

Pour $T_{\mathrm{c}}-T>1{ }^{\circ} \mathrm{C}$, le spectre est un pic aigu dont il est facile de déterminer la fréquence $v^{(a)}$.

Pour $T_{\mathrm{c}}-T<1^{\circ} \mathrm{C}$, le spectre s'étale, le rapport signal sur bruit est moins bon. Pour avoir une bonne détermination de $v^{(a)}$ et $\Delta v^{(a)}$, nous avons utilisé un grand nombre de spectres expérimentaux pour chaque valeur de $\left(T_{\mathrm{c}}-T\right)$ et de $q$ - généralement 6 et fait la moyenne sur les différentes valeurs mesurées. Ceci permet aussi de moyenner les éventuelles dérives de l'analyseur d'onde lorsque les fréquences mesurées sont basses $\left(15,5 \mathrm{~Hz}\right.$ pour $q=150 \mathrm{~cm}^{-1}$ et $T_{\mathrm{c}}-T=0,024^{\circ} \mathrm{C}$ ). Afin d'améliorer la précision, dans tous les cas où la largeur du spectre 


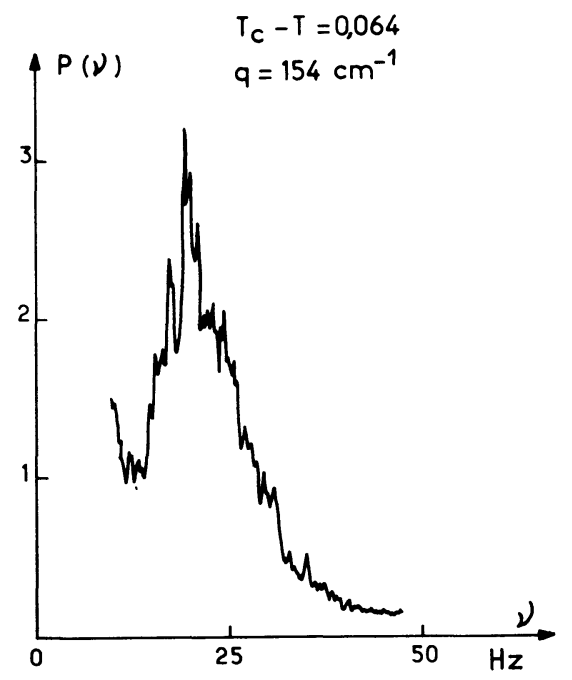

a
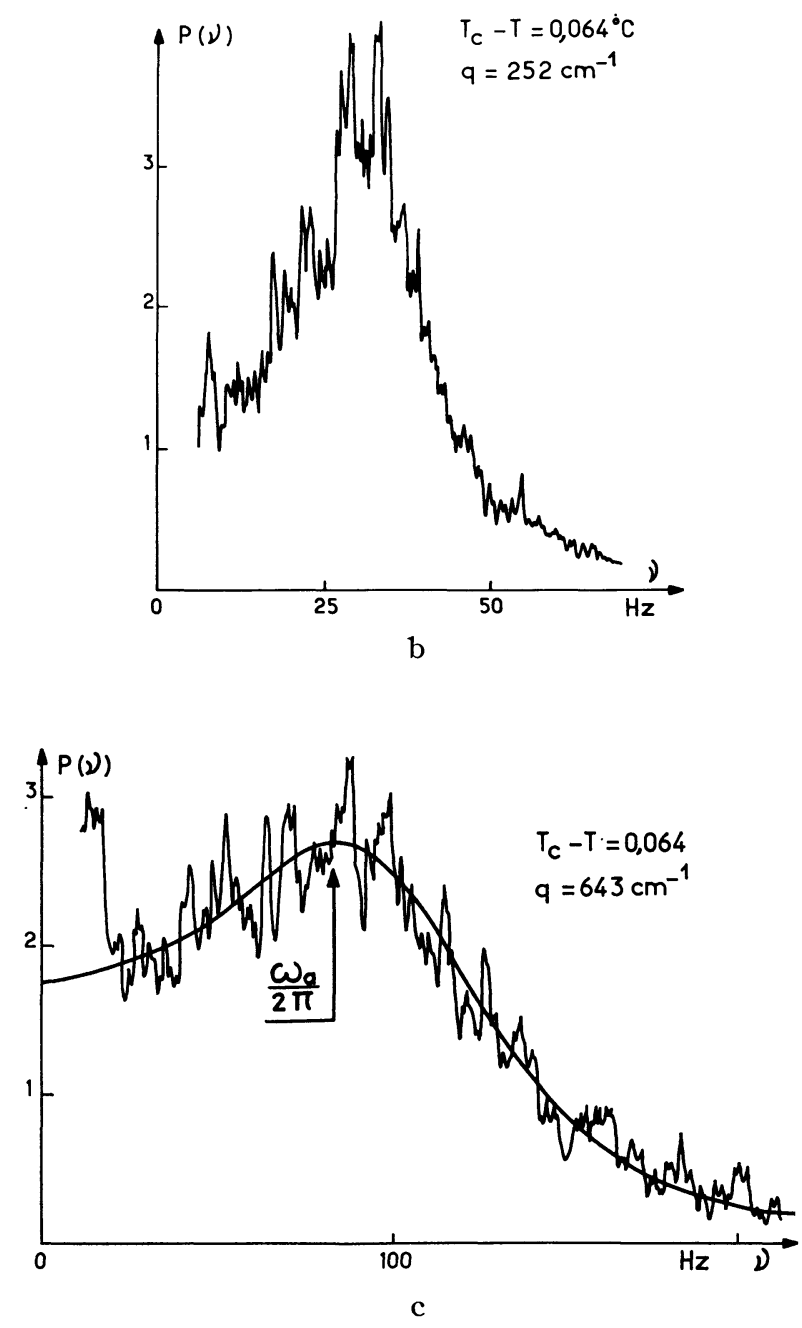

avait un sens, nous avons recherché la courbe théorique $P_{q}(\nu)$ qui offrait le meilleur accord avec la courbe expérimentale (fig. $4 \mathrm{c}$ ). Très près du point critique $\left(T_{\mathrm{c}}-T \leqslant 0,044^{\circ} \mathrm{G}\right)$, les spectres présentent en plus un maximum à la fréquence zéro qui est dû soit à la diffusion Rayleigh, soit à la diffusion sur les courants de convection créés par le faisceau laser. Afin de réduire ces derniers, la puissance laser a été abaissée à moins de $0,2 \mathrm{~mW} / \mathrm{cm}^{2}$ pour ces mesures. Pour tenir compte de cet effet parasite, nous avons recherché quel était le spectre théorique $P_{q}(\nu)+A /\left(\nu^{2}+v_{1}^{2}\right)$ qui nous offrait le meilleur accord avec le spectre expérimental étudié ( fig. $4 \mathrm{~d}$ ).

En conclusion, ces mesures fournissent $\nu_{a}$ et éventuellement $\Delta v_{a}$ pour chaque valeur $T$ de la température. La valeur de $T_{\mathrm{c}}$ a été déterminée une fois pour toute en recherchant la température à laquelle l'absorption du faisceau laser traversant la cellule était maximum. En tenant compte d'éventuelles dérives du thermomètre, $T_{\mathrm{c}}-T$ est ainsi déterminé à mieux de $0,002^{\circ} \mathrm{C}$ près.

B) A partir de $v_{a}(q, T)$ (et $\left.\Delta v_{a}(q, T)\right)$, on peut déduire $\alpha_{q}$ (et $\left.\eta_{\mathrm{c}}\right)$. Nous distinguerons deux cas suivant que l'on est relativement loin ou près de $T_{\mathrm{c}}$ :

1) $T_{\mathrm{c}}-T \geqslant 0,2^{\circ} \mathrm{C}$, on calcule alors :

$$
S_{i}^{a}=2 \pi v_{a} \frac{\rho+\rho^{\prime}}{2\left(\eta+\eta^{\prime}\right) q^{2}}
$$

$\rho$ et $\rho$ ' sont donnés par des tables expérimentales [12], $\eta$ est déduit de la courbe de la figure 1 (chap. II). On tire $\alpha_{q}$ de la résolution numérique des équations (2) et (3). On recherche $S_{i}$ et $S_{r}$ satisfaisant (2) et donnant une valeur de $y$ calculée par l'équation (3) qui soit réelle.

De la valeur de $y$ :

$$
y=\frac{\alpha_{q}\left(p+p^{\prime}\right)}{4\left(\eta+\eta^{\prime}\right)^{2} q}
$$

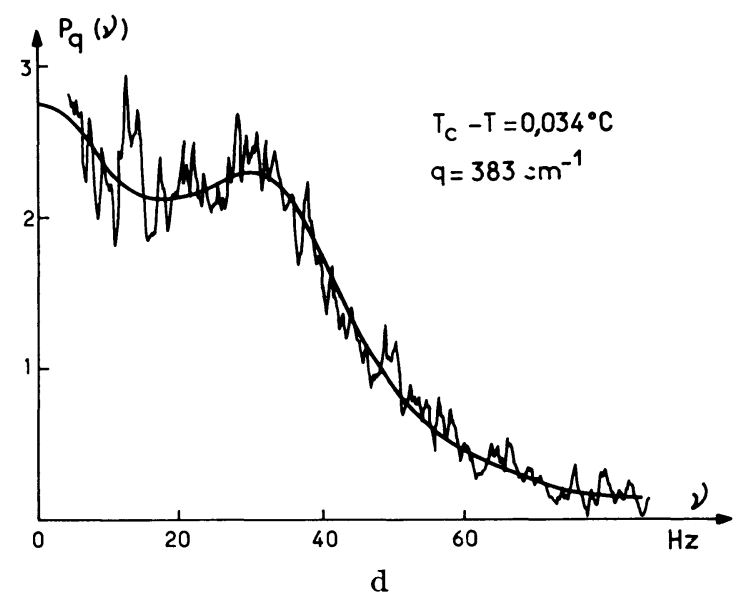

FiG. 4. - Spectres expérimentaux de la lumière diffusée par les ondes de surface ainsi que les spectres théoriques correspondants lorsque l'amortissement est grand (c et d). 
on déduit celle de :

$$
\alpha_{q}=\alpha+\frac{\Delta p \cdot g}{q^{2}}
$$

Dans le domaine de température considéré ici, le second terme (gravité) est toujours très petit devant le premier (capillarité). Il représente une correction au plus de quelque $10^{-2}$, aisément calculable.

2) $T_{\mathrm{c}}-T \leqslant 0,2^{\circ} \mathrm{C}$, nous avons utilisé l'équation (4) plus simple à résoudre et nous avons vérifié que pour $T_{\mathrm{c}}-T=0,2^{\circ} \mathrm{C}$ et $T_{\mathrm{c}}-T=0,5{ }^{\circ} \mathrm{C}$ nous obtenions bien les mêmes valeurs de $\alpha_{q}$ que par la méthode précédente.

On calcule :

$$
S_{i}^{a}=2 \pi v_{a} \frac{\rho_{\mathrm{c}}}{2 \eta_{\mathrm{c}} q^{2}}
$$

et éventuellement :

$$
S_{r}^{a}=\pi \Delta \nu_{a} \frac{\rho_{\mathrm{c}}}{2 \eta_{\mathrm{c}} q^{2}}
$$

avec $\rho_{\mathrm{c}}=0,464 \mathrm{~g} / \mathrm{cm}^{3}$ et $\eta_{\mathrm{c}}=350 \times 10^{-6}$ poise, valeur admise précédemment (chap. II). Cette valeur est en bon accord avec la forme des spectres expérimentaux que nous avons obtenus. La figure $4 \mathrm{~d}$ montre un spectre expérimental pour $T_{\mathrm{c}}-T=0,034^{\circ} \mathrm{C}$ et le spectre théorique correspondant à la valeur $\eta_{\mathrm{c}}=350 \times 10^{-6}$ poise. Nous pouvons en conclure qu'à $0,034^{\circ} \mathrm{G}$ du point critique la viscosité ne présente pas d'anomalie importante : si elle en présente une, elle est inférieure à $15 \%$; nous en déduisons aussi :

$$
\eta_{\mathrm{c}}=350 \pm 50 \times 10^{-6} \text { poise. }
$$

Les valeurs $S_{i}^{a}$ et $S_{r}^{a}$ ainsi calculées, reportées sur les courbes de la figure 2 , fournissent chacune une valeur de $y_{\mathrm{c}}: y_{\mathrm{c}}^{i}$ et $y_{\mathrm{c}}^{r}$. Il résulte de l'accord entre les formes spectrales théoriques et expérimentales, qu'avec la valeur $\eta_{\mathrm{c}}=350 \pm 50 \times 10^{-6}$ poise précédente, on obtient :

$$
y_{\mathrm{c}}^{i}=y_{\mathrm{c}}^{r}=y_{\mathrm{r}}
$$

aux erreurs de mesure près.

On en déduit :

$$
\alpha_{q}=\alpha+\frac{\Delta \rho \cdot g}{q^{2}}=y_{\mathrm{c}} \frac{8 \eta_{\mathrm{c}}^{2} q}{\rho_{\mathrm{c}}} .
$$

Pour chaque valeur de $T_{\mathrm{c}}-T$, on trouve la droite $\alpha_{q}$ en fonction de $1 / q^{2}$ (fig. 5). L'ordonnée à l'origine de la droite donne $\alpha$, tandis que de sa pente on déduit $\Delta \rho$. Cette pente n'est mesurable qu'à l'approche de $T_{\mathrm{c}}$. On sait en effet que $\alpha$ et $\Delta \rho$ varient comme $\left(T_{\mathrm{c}}-T\right)^{\mu}$ et $\left(T_{\mathrm{c}}-T\right)^{\beta}$ avec $\mu / \beta \approx 4$. La détermination de $\Delta \rho$ est seulement possible lorsque les forces de gravité ne sont plus négligeables devant les forces de capillarité.

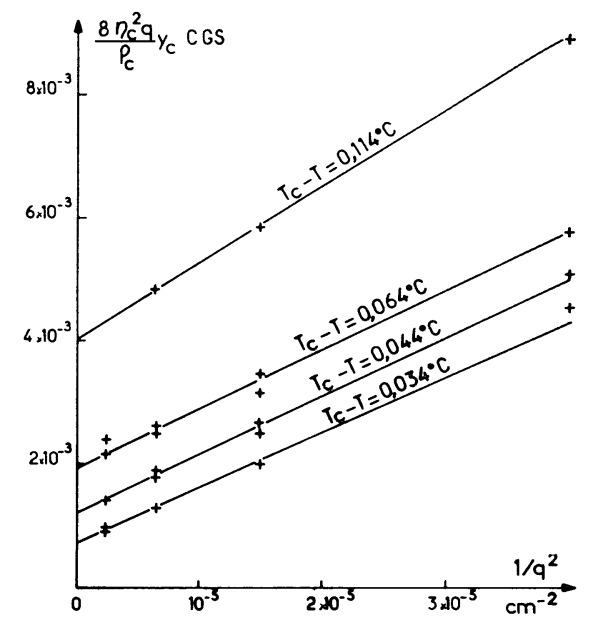

FIG. 5. $-\alpha_{q}$ en fonction de $q^{-2}$ pour diverses valeurs de $T_{\mathrm{e}}-T$. $\alpha$ est donné par l'ordonnée à l'origine, tandis que $\Delta p$ est déduit de la pente.

C) La mesure de la tension superficielle a été faite dans le domaine de température :

$$
0,034 \leqslant T_{\mathrm{c}}-T \leqslant 31^{\circ} \mathrm{C}
$$

soit sur trois décades.

La tension superficielle varie sur quatre décades :

$$
8 \times 10^{-4} \leqslant \alpha \leqslant 6 \text { dynes } / \mathrm{cm} .
$$

Sur la figure 6 , nous avons porté en échelles logarithmiques $\alpha$ en fonction de $T_{\mathrm{c}}-T$. Les points s'alignent sur une droite de pente 1,253 , ce qui nous conduit à la relation :

$$
\alpha=\alpha_{0}\left(1-\frac{T}{T_{\mathrm{c}}}\right)^{\mu}
$$

avec, dans le domaine de température cité plus haut :

$$
\mu=1,253 \pm 0,01 ; \quad \alpha_{0}=17,9 \pm 1,2 \text { dynes } / \mathrm{cm} \text {. }
$$

L'incertitude sur $\mu$ due aux erreurs statistiques est $\pm 0,005 \mathrm{~d}$ 'après la dispersion des points expérimentaux; celle due à l'erreur sur la viscosité est $\pm 0,002$ si nous admettons la valeur $\eta_{\mathrm{c}}$ déduite de la figure 1 (chap. II) et $\pm 0,0035$ si nous admettons la valeur déduite de nos mesures. Enfin, nous devons tenir compte d'une troisième cause d'erreur sur $\mu$, celle qui provient de la mesure de $q$; elle peut être estimée à $\pm 0,003$. L'incertitude totale sur $\mu$ est donc $\pm 0,01$ si nous admettons la valeur de la viscosité donnée dans la référence [6] et $\pm 0,012$ si nous utilisons la viscosité déduite de nos mesures.

Différents auteurs [14] ont déterminé de manière précise la variation de $\Delta \rho$ en fonction de $T_{\mathrm{c}}-T$ :

$$
\Delta p=\left(T_{\mathrm{c}}-T\right)^{\beta} \text { avec } \beta=0,34 .
$$

Les mesures de $\Delta \rho$ que nous avons faites s'étendent dans un étroit domaine de température :

$$
0,024 \leqslant T_{\mathrm{c}}-T \leqslant 0,1^{\circ} \mathrm{C} .
$$


Elles n'ont pas pour but d'étudier $\Delta \rho$. Nous avons seulement voulu vérifier que nos mesures coïncident avec les mesures faites par d'autres auteurs et justifier ainsi la validité de notre méthode de dépouillement assez complexe. Les résultats expérimentaux que nous avons obtenus pour $\Delta \rho$ sont portés sur la figure 6 . On

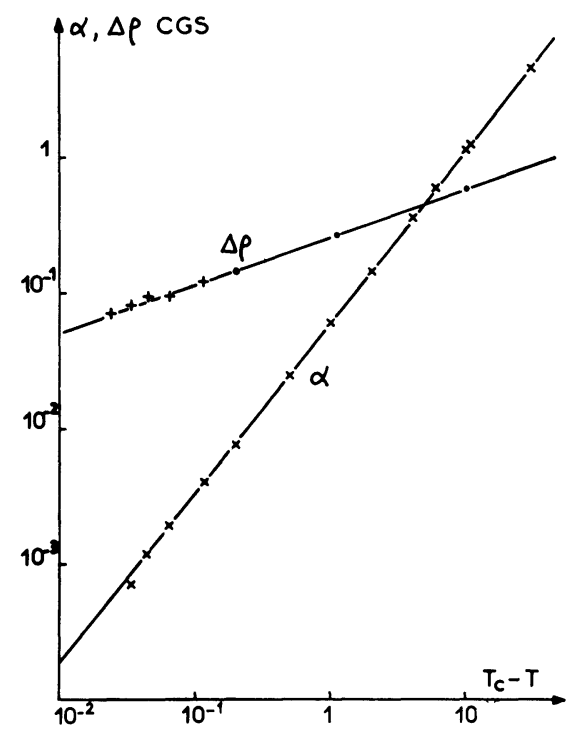

FIG. 6. - La tension superficielle $\alpha$ en fonction de $T_{\mathrm{e}}-T$ en échelle logarithmique (la pente de la droite tracée est 1,25 ) et l'écart de masse spécifique entre phases. Les trois points sur la droite $\Delta p$ sont trois valeurs données par les tables de valeurs expérimentales (référence [12]).

vérifie qu'ils se placent bien sur une droite de pente 0,34 passant par les points suivants tirés de tables de données expérimentales [12] :

$$
\begin{aligned}
& T_{\mathrm{c}}-T=10,1{ }^{\circ} \mathrm{G} ; \quad \Delta \rho=0,563 \mathrm{~g} / \mathrm{cm}^{3} \\
& T_{\mathrm{c}}-T=1,1^{\circ} \mathrm{C} ; \quad \Delta \rho=0,265 \mathrm{~g} / \mathrm{cm}^{3} \\
& T_{\mathrm{c}}-T=0,1{ }^{\circ} \mathrm{C} ; \quad \Delta \rho=0,141 \mathrm{~g} / \mathrm{cm}^{3} .
\end{aligned}
$$

La tension superficielle n'a pas pu être mesurée pour $T_{\mathrm{c}}-T<0,034^{\circ} \mathrm{C}$. Il paraît plausible d'admettre que la limitation à nos mesures provient du phénomène suivant : à l'approche de $T_{\mathrm{c}}$, l'épaisseur de la zone de transition entre le liquide et le gaz devient de l'ordre de grandeur de la longueur d'onde optique utilisée (6 $328 \AA$ ). Le pouvoir réflecteur de la surface décroît alors brusquement. L'opalescence de volume domine alors l'opalescence de surface [3]. L'utilisation d'un faisceau laser infrarouge, proposée par Papoular [3], pour pallier ces difficultés dans le cas d'un mélange critique de démixtion, ne semble pas pouvoir être appliquée à un fluide pur, dans nos conditions d'expérience. D'une part, à angle de diffusion égal, le vecteur d'onde $q$ est plus petit et par suite les forces de gravité domineraient les forces de capillarité, d'autre part l'absorption d'un tel faisceau serait certainement plus grande et provoquerait des courants de convection plus forts d'autant plus que l'expérience nécessiterait un faisceau plus intense pour compenser le faible rendement des détecteurs pour ces longueurs d'ondes.

Pour $T_{\mathrm{c}}-T$ grand, la courbure de l'interface devient importante par suite des valeurs élevées de la tension superficielle et du petit diamètre interne de la cellule utilisée $(2 \mathrm{~cm})$. Ceci a limité nos mesures à $T_{\mathrm{c}}-T=31^{\circ} \mathrm{C}$.

V. Conclusion. - La méthode employée a permis la mesure de la tension superficielle pour des valeurs de celle-ci inférieures à $10^{-3}$ dyne $/ \mathrm{cm}$. La grande étendue de la zone de mesure (quatre décades sur la tension superficielle), très supérieure à celle autorisée par la méthode classique des tubes capillaires, a permis une bonne précision sur la valeur de l'exposant $\mu$. Nous donnons dans le tableau I à titre de comparaison les résultats obtenus sur divers fluides simples par différents auteurs à l'aide de tubes capillaires.

Sur le plan théorique, Widom [17] a reformulé la théorie de la tension superficielle d'un fluide au voisinage du point critique en faisant l'hypothèse que l'épaisseur de l'interface varie comme la longueur de corrélation. Entre les exposants critiques $\beta, \gamma^{\prime}$, $\nu$ et $\mu$ (relatifs à la différence de masse spécifique, la compressibilité au-dessous de $T_{\mathrm{c}}$, la longueur de corrélation et la tension superficielle), il obtient ainsi la relation :

$$
2 \beta+\gamma^{\prime}=\mu+\nu
$$

à laquelle on peut adjoindre une seconde reliant $\gamma$ (exposant critique relatif à la compressibilité au-dessus de $T_{\mathrm{c}}$ ) et $\nu[18]$ :

$$
\gamma=\left(2-\eta_{\mathrm{F}}\right) \nu
$$

\section{TABLEAU I}

$\begin{array}{lccc} & & \text { Domaine EXPLORÉ } & \\ & \mu & \varepsilon=1-T / T_{\mathrm{c}} & \text { RÉfÉRENCE } \\ & - & - & - \\ \mathrm{A} & 1,281 & 0,4-4 \times 10^{-2} & {[15]} \\ \mathrm{N}_{2} & 1,247 & 0,5-2 \times 10^{-2} & {[15]} \\ \mathrm{Xe} & 1,287 \pm 0,017 & 0,4-1,6 \times 10^{-2} & {[16]} \\ \mathrm{CO}_{2} & 1,253 \pm 0,01 & 0,1-1 \times 10^{-4} & \text { Présent travail }\end{array}$


$\left(\eta_{\mathrm{F}}\right.$ étant l'exposant caractéristique de la fonction de distribution des paires ou fonction de corrélation en volume $\left.g(r)-1 \propto \mathrm{e}^{-r / \xi} / r^{1+n_{\mathrm{F}}}\right)$.

Les valeurs les plus probables des exposants qui figurent ci-dessus semblent être à l'heure actuelle [19] :

$$
\beta=0,345 ; \quad \gamma^{\prime} \approx 1,2 ; \quad \gamma \approx 1,2 ; \quad \eta_{\mathrm{F}} \lesssim 0,1
$$

conduisant à $2 \beta+\gamma^{\prime}-\frac{\gamma}{2-\eta}=1,26$ en accord raisonnable avec la valeur expérimentale obtenue pour $\mu$, étant donné l'incertitude très appréciable qui règne sur $\gamma$ et $\gamma^{\prime}(10 \%$ au moins) [20].

Appendice. - Si le fluide est compressible, il peut exister un certain couplage entre les fluctuations de surface et de densité; nous allons montrer que, même à l'approche du point critique (où la compressibilité diverge), ce couplage demeure négligeable tant que les forces de capillarité l'emportent sur les forces de gravité et tant que le régime reste hydrodynamique $(1 / q\rangle\rangle$ portée des corrélations spatiales).

Soient $\rho_{0}$ la masse spécifique moyenne dufluide et $\delta \rho$ ses fluctuations. Soit $\mathbf{V}+\delta \mathbf{V}$ la vitesse du fluide, $\mathbf{V}$ représentant la vitesse associée au fluide incompressible de masse spécifique $p_{0}$.

L'équation de conservation de la masse :

$$
\operatorname{div}\left(\rho_{0}+\delta \rho\right)(\mathbf{v}+\delta \mathbf{V})+\frac{\partial\left(\rho_{0}+\delta \rho\right)}{\partial t}=0
$$

conduit à la relation :

$$
\rho^{0} \mathbf{q} \delta \mathbf{V}_{q \omega}+\delta \rho_{q \omega}(\mathbf{q} \mathbf{V}-\omega)=0
$$

si l'on prend les transformées de Fourier spatiale et temporelle des deux membres :

$$
\begin{aligned}
\delta \mathbf{V}(r, t) & =\int \delta \mathbf{V}_{q \omega} \mathrm{e}^{i \mathbf{q} \cdot \mathbf{r}-i \omega t} \frac{\mathscr{V}}{(2 \pi)^{3}} \mathrm{~d}^{3} \mathbf{q} \mathrm{d} \omega \\
\rho(r, t) & =\int \delta \rho_{q \omega} \mathrm{e}^{i \mathbf{q} \cdot \mathbf{r}-i \omega t} \frac{\mathscr{V}}{(2 \pi)^{3}} \mathrm{~d}^{3} q \mathrm{~d} \omega
\end{aligned}
$$

et si l'on néglige $\delta \rho / \rho_{0}$ devant 1 .

Dans l'équation (2), nous pouvons négliger $\mathbf{q V}$ devant $\omega$ (ceci revient à négliger l'amplitude des fluctuations devant leur longueur d'onde).

On obtient alors :

$$
\delta V_{q \omega} \approx \frac{\omega}{q} \frac{\delta \rho_{q \omega}}{\rho_{0}} .
$$

Cette quantité est à comparer à :

$$
V_{q \omega} \approx \omega \zeta_{q \omega}
$$

( $\zeta_{q \omega}$ est l'amplitude de l'onde de surface de vecteur $q$ et de pulsation $\omega$ ).

Nous évaluons le rapport :

$$
c(\omega)=\frac{\overline{\left|\delta V_{q \omega}\right|^{2}}}{\overline{\left|V_{q \omega}\right|^{2}}}=\frac{1}{q^{2} \overline{\left|\zeta_{q \omega}\right|^{2}}} \frac{\overline{\left|\delta \rho_{q \omega}\right|^{2}}}{\rho_{0}^{2}} .
$$

Tant que $c$ est très petit devant l'unité, nous pourrons dire que les fluctuations de surface et les fluctuations de densité en volume sont pratiquement découplées. Les quantités $\delta \rho_{q \omega}$ et $\zeta_{q \omega}$ figurant dans l'expression (3) sont celles que l'on calcule en négligeant tout couplage :

$$
\begin{aligned}
& \overline{\left|\delta \rho_{q \omega}\right|^{2}}=\overline{\left|\delta \rho_{q}\right|^{2}} S_{\rho, q}(\omega) \\
& \overline{\left|\zeta_{q \omega}\right|^{2}}=\overline{\left|\zeta_{q}\right|^{2}} S_{\zeta, q}(\omega)
\end{aligned}
$$

$S_{\rho, q}(\omega)$ et $S_{\zeta, q}(\omega)$ sont les densités spectrales des fluctuations thermiques de densité et de coordonnée verticale des points de la surface, normalisés de telle sorte que :

$$
\int S_{\rho, q}(\omega) \mathrm{d} \omega=1 ; \quad \int S_{\zeta, q}(\omega) \mathrm{d} \omega=1 .
$$

La mécanique statistique permet de plus d'évaluer :

$$
\begin{aligned}
& \overline{\left|\delta \rho_{q}\right|^{2}}=-\rho_{0}^{2} k_{\mathrm{B}} T\left(\frac{\partial \rho}{\partial P}\right)_{T} \\
& \overline{\left|\zeta_{q}\right|^{2}}=\frac{k_{\mathrm{B}} T}{\left(\alpha q^{2}+\Delta \rho g\right) A} .
\end{aligned}
$$

On en déduit :

$$
c(\omega)=\left(\frac{\partial \rho}{\partial P}\right)_{T} \cdot\left(\alpha+\frac{\Delta \rho g}{q^{2}}\right) A \cdot \frac{S_{\rho, q}(\omega)}{S_{\zeta, q}(\omega)} .
$$

Or, au voisinage du point critique :

$$
\begin{aligned}
& \left(\frac{\partial \rho}{\partial P}\right)_{T} \propto \frac{1}{\left(T_{\mathrm{c}}-T\right)^{\gamma}} \quad \text { avec } \quad \gamma=1,2 \\
& \alpha \propto\left(T_{\mathrm{c}}-T\right)^{\mu} \text { avec } \mu=1,2 \\
& \Delta \rho \propto\left(T_{\mathrm{c}}-T\right)^{\beta} \text { avec } \beta=0,34 \text {. }
\end{aligned}
$$

Les exposants $\gamma$ et $\mu$ étant très voisins, la valeur du produit $\left(\frac{\partial \rho}{\partial P}\right)_{T} . \alpha_{q}$ demeure pratiquement constante $\left(\approx 2 \times 10^{-8}\right)$ à l'approche de $T_{\mathrm{c}}$, tant que les forces de capillarité demeurent prépondérantes.

A l'approche de $T_{\mathrm{c}}$, il paraît justifié d'écrire $S_{\rho, q}(\omega)$ sous la forme $\delta(\omega)$, tenant compte du fait que la raie Rayleigh comprend la presque totalité de l'intensité (les composantes Brillouin sont relativement très peu intenses) et a une largeur très étroite devant la résolution $3 \mathrm{~Hz}$ de l'analyseur d'onde (d'après [21] pour $T_{\mathrm{c}}-T=0,044^{\circ} \mathrm{C} ; q=380 \mathrm{~cm}^{-1}$, on attend une largeur de 0,2 cycle). Par suite, en intégrant le rapport $S_{\rho, q}(\omega) / S_{\zeta, q}(\omega)$ sur un intervalle égal à la résolution de notre spectromètre, nous obtenons pratiquement 0 si $\omega \neq 0$ et :

$c(0)=\left(\frac{\partial \rho}{\partial P}\right)_{T} \cdot\left(\alpha+\frac{\Delta \rho g}{q^{2}}\right) A \cdot \frac{1}{S_{\zeta, q}(0)} \quad$ si $\quad \omega=0$.

A l'aide de cette expression, nous pouvons aisément vérifier que la condition $c(0) \ll 10^{-5}$ était satisfaite dans tous les cas étudiés expérimentalement. Il est donc justifié de considérer que les fluctuations de densité et de surface sont entièrement découplées comme pour un fluide incompressible. 
Ge résultat peut être obtenu d'une manière moins rigoureuse mais plus parlante en remarquant que la compressibilité du fluide n'intervient pas dans la propagation des ondes de surface si celles-ci sont complètement découplées des ondes sonores dans le milieu. Ce découplage est réalisé lorsque les vitesses de propagations $V_{\text {surf. }}$ et $V_{\text {vol. }}$ de ces ondes sont très différentes. Pour un amortissement faible (ce qui est mal réalisé près de $\left.T_{\mathrm{c}}\right)$ :

$$
V_{\text {surf. }} / V_{\text {vol. }}=\sqrt{\alpha \chi q} \quad(\chi \text { compressibilité }) .
$$

Nous avons vu précédemment que $\alpha \chi$ varie de manière négligeable avec $T_{\mathrm{c}}-T$. La relation $V_{\text {surf. }} / V_{\text {vol. }} \ll 1$ valable loin de $T_{\text {c }}$ reste donc valable près de $T_{\mathrm{c}}$.

Je tiens à remercier M. Papoular pour cette dernière remarque ainsi que pour toutes les discussions fructueuses que $\mathrm{j}$ 'ai eues avec lui.

Je remercie Mme M. A. Bouchiat pour toute l'aide et les conseils qu'elle m'a donnés au cours de ce travail.

\section{BIBLIOGRAPHIE}

[1] Cruchon (D.), Meunier (J.) et Bouchiat (M. A.), C. R. Acad. Sci. Paris, 1968, 267, 32.

[2] Meunier (J.), Cruchon (D.) et Bouchiat (M. A.), C. R. Acad. Sci. Paris, 1969, 268, 422.

[3] Papoular (M.), C. R. Acad. Sci. Paris, 1968, 266, 592.

[4] Meunier (J.), Cruchon (D.) et Bouchiat (M. A.), C. R. Acad. Sci. Paris, 1969, 268, 92.

[5] Papoular (M.), J. Physique, 1968, 29, 81.

[6] Kestin (J.), Whitelaw (J. M.) et ZiEN (T. F.), Physica, 1964, 30, 161.

[7] Senghers (J. V.), Critical Phenomena, Proceedings of a conference, Washington, D.C., 1965.

[8] RAYLEIGH (J. W.), Scientific Papers, V, 322, 388.

[9] Mandelstam (L. I.), Ann. Physik, 1913, 41, 609.

[10] FABEIINSKII (I. L.), Molecular Scattering of Light, New York, 1968.

[11] Bouchiat (M. A.), Meunier (J.) et Brossel (J.), C. R. Acad. Sci. Paris, 1968, 266, 255.

[12] International critical tables of numerical data, vol. III, MacGraw-Hill Book Company, 1928.
[13] Temperley (H. N. V.), Rowlinson (J. S.) et Rushbrooke (G. S.), Physics of Simple Liquids, North-Holland, 1968, 558.

[14] Micheis (A.), Bifaise (B.) et Micheis (C.), Proc. Roy. Soc., 1937, A 160, 358.

I,ORENZTSEN (H. L.), Acta Chem. Scand., 1953, 7, 1336. Statistical Mechanics of Equilibrium and Non-Equilibrium, Ed. J. Meixner (Amsterdam, North-Holland).

[15] Stansfield (D.), Proc. Phys. Soc., 1958, 72, 854.

[16] Smith (B. L.), Gardner (P. R.) et Parker (E. H. C.), J. Chem. Phys., 1967, 47, 1148.

[17] Widom (B.), J. Chem. Phys., 1965, 43, 3892.

[18] Fisher (M. E.), J. Math. Phys., 1964, 5, 944.

[19] EgeistafF (P. A.) et RING (J. W.), in Physics of Simple Liquids, edited by Temperley (H. N. V.), Rowlinson (J. S.) and Rushbrooke (G. S.), NorthHolland Publishing Cy, 1968, ch. 7.

[20] Hellek (P.), Rept. Progr. Phys., 1967, 30, 731.

[21] Swinney (H. L.) et Cummins (H. Z.), Phys. Rev., 1968, 171, 152. 